\title{
On Concentration of Positive Bound States of Nonlinear Schrödinger Equations
}

\section{Xuefeng Wang ${ }^{\star}$}

Department of Mathematics, Tulane University, New Orleans, LA 70118, USA

Received April 8, 1992; in revised form August 31, 1992

Abstract. We study the concentration behavior of positive bound states of the nonlinear Schrödinger equation

$$
i h \frac{\partial \psi}{\partial t}=\frac{-h^{2}}{2 m} \Delta \psi+V(x) \psi-\gamma|\psi|^{p-1} \psi .
$$

Under certain condition on $V$, we show that positive ground state solutions must concentrate at global minimum points of $V$ as $h \rightarrow 0^{+}$; moreover, a point at which a sequence of positive bound states concentrates must be a critical point of $V$. In case that $V$ is radial, we prove that the positive radial solutions with least energy among all nontrivial radial solutions must concentrate at the origin as $h \rightarrow 0^{+}$.

\section{Section 1. Introduction and Description of Main Results}

Of concern are standing wave solutions of the following nonlinear Schrödinger equations:

$$
i h \frac{\partial \psi}{\partial t}=\frac{-h^{2}}{2 m} \Delta \psi+V(x) \psi-\gamma|\psi|^{p-1} \psi \quad \text { with } x \in \mathbb{R}^{n}
$$

i.e., solutions of the form

$$
\psi(x, t)=\exp (i E t / h) u(x)
$$

where $h, m, \gamma$ and $p$ are positive constants, $p>1, E \in \mathbb{R}, V$ is real and belongs to $C^{1}\left(\mathbb{R}^{n}\right)$ and $u$ is real. In [FW], Floer and Weinstein proved for small $h>0$ (and for $p=3, n=1$ ) the existence of standing wave solutions concentrating at each given nondegenerate critical point of the potential $V$, under the condition that $V$ is bounded. In $\left[\mathrm{O}_{1}, \mathrm{O}_{3}\right]$, Oh generalized this result and obtained for small $h>0$ the existence of multi-lump standing wave solutions with $u$ in (1.2) being positive and

\footnotetext{
* Research supported in part by NSF Grant DMS-9105172.
} 
concentrating at any given finite collection of nondegenerate critical points of $V$, under the condition $n \geqq 1, \quad 1<p<\frac{n+2}{(n-2)^{+}}$(we use the convention: $\frac{n+2}{(n-2)^{+}}=\infty$ when $\left.n=1,2\right)$, and $V \in(V)_{a}$ (namely, either $V \equiv a$ or $V(x)>a$ and $\left.(V-a)^{-1 / 2} \in \operatorname{Lip}\left(\mathbb{R}^{n}\right)\right)$. The arguments in these papers are based on the Lyapunov-Schmidt reduction.

Substituting (1.2) into (1.1) and assuming without loss of generality, $2 m=1$ and $\gamma=1$, one has

$$
h^{2} \Delta u-(V(x)-E) u+|u|^{p-1} u=0, \quad x \in \mathbb{R}^{n} .
$$

Throughout this paper, we shall assume $1<p<\frac{n+2}{(n-2)^{+}}$and $V$ is bounded below. A suitable choice of $E$ makes $V-E$ bounded below from zero. Thus, without loss of generality, we shall assume throughout this paper that $E=0$ and $V$ is bounded below by a positive constant. Now the equation for $u$ can be rewritten as

$$
h^{2} \Delta u-V(x) u+|u|^{p-1} u=0, \quad x \in \mathbb{R}^{n},
$$

or

$$
\Delta v-V(h x) v+|v|^{p-1} v=0, \quad x \in \mathbb{R}^{n},
$$

where $v(x)=u(h x)$ and inf $V>0$. The existence of solutions of (1.3) (or (1.4)) and its various generalizations has long been studied extensively (mostly by variational methods). The interested readers may consult, in addition to the papers mentioned below, the survey articles $\left[\mathrm{L}_{1}\right]$ and $[\mathrm{N}]$ and references listed therein. Most of the results provide existence of solutions for arbitrary $h>0$. Several papers deal with existence of ground states, i.e., in case of (1.3), solutions with least "energy"

$$
\frac{1}{2} \int_{\mathbb{R}^{n}}\left(h^{2}|\nabla u|^{2}+V u^{2}\right) d x-\frac{1}{p+1} \int_{\mathbb{R}^{n}}|u|^{p+1} d x
$$

among all nontrivial $H^{1}\left(\mathbb{R}^{n}\right)$ solutions of (1.3). Here we mention two papers that can be directly applied to (1.3). In [DN], Ding and Ni proved among other things, that if $V$ is, roughly speaking, ultimately increasing along $n$ independent directions and "almost symmetric", then for $h>0,(1.3)$ (or (1.4)) has a positive ground state (it was not stated in [DN] that the solution is a ground state, yet it is possible to check the solution is one). Another general result of [DN] implies that if $n \geqq 2$ and $V$ is radial, then for every $h>0,(1.3)$ (or (1.4)) has a radial positive solution with least energy among all nontrivial $H^{1}\left(\mathbb{R}^{n}\right)$ radial solutions. (We shall call them radial ground states.) Recently, Rabinowitz [R] showed that (1.3) (or (1.4)) has a positive ground state for every $h>0$ if $\liminf _{x \rightarrow \infty} V(x)=\sup V$ or for small $h>0$ if $\lim \inf _{x \rightarrow \infty} V(x)>\inf V$. In both [DN] and [R], more general nonlinearity was treated, and the Mountain-Pass arguments were used.

In this paper, motivated by a question in [R], we study the concentration behavior of positive bound states (i.e., solutions with finite energy) of (1.3) as $h \rightarrow 0^{+}$. Concerning positive ground states, we obtain what can be roughly described as follows. If lim $\inf _{x \rightarrow \infty} V(x)>\inf V$, any sequence of positive ground states of (1.3) contains a subsequence concentrating at a global minimum point of $V$ as $h \rightarrow 0^{+}$. (In particular, if the global minimum point of $V$ is unique, then all positive 
ground states concentrate at that point as $h \rightarrow 0^{+}$.) On the other hand, if we only know a sequence of ground states $u_{h_{k}}$ exists and there is a sequence of local maximum points moving toward a certain point $x_{0}$ as $h_{k} \rightarrow 0^{+}$, then $x_{0}$ is a global minimum point of $V$ and $\left\{u_{h_{k}}\right\}$ concentrates at $x_{0}$ as $h_{k} \rightarrow 0^{+}$. (Thus if $V$ has no global minimum, the positive ground states, if any, do not concentrate.) As for radial positive ground states of (1.3), we prove that if $n \geqq 2$, all of them concentrate at the origin as $h \rightarrow 0^{+}$. For general positive bound states, we show that under the condition $|\nabla V(x)|$ $=O\left(e^{a|x|}\right)$ near $x=\infty$ for some $a>0$, a point at which a sequence of positive bound states of (1.3) concentrates must be a critical point of $V$ (this result is converse to those of Floer, Weinstein and $\mathrm{Oh}$ ). For the precise statements of our results, see Theorem 2.1, Theorem 2.3, Theorem 2.5 and Theorem 3.1.

We should mention a recent work of $\mathrm{Ni}$ and Takagi [NT] concerning the asymptotic behavior of least energy solutions of the Neumann problem for (1.3) (with $V \equiv 1$ but more general nonlinear term) on a bounded domain $\Omega$. They proved in [NT] that positive least energy solutions must exhibit "point-condensation" character on the boundary $\partial \Omega$. More recently, they announced that they have proved that these solutions concentrate at a point on $\partial \Omega$ where the maximum mean curvature of $\partial \Omega$ is achieved. Some of our arguments in Sect. 2 are inspired by [NT].

Finally, we wish to take this opportunity to point out that the main results in $[\mathrm{FW}]$ and $\left[\mathrm{O}_{1}, \mathrm{O}_{3}\right]$ are still true as long as $V$ is bounded - we do not need $V \in \mathrm{Kato}$ class $(V)_{a}$ in this case. (So $V$ can be highly oscillatory at $\infty$ as in the case of $V(x)=\sin |x|^{2}$ or $\sin e^{|x|}$.) The reason is that $V \in(V)_{a}$ is only needed in these papers to show

$$
\|(-\Delta+V(h x)-E) u\|_{L^{2}\left(\mathbb{R}^{n}\right)} \geqq \lambda\|u\|_{H^{2}\left(\mathbb{R}^{n}\right)},
$$

where $\lambda>0$ is independent of $h>0, V(h x)-E \geqq \delta>0$ in $\mathbb{R}^{n}$ (see (2.9) in [FW], (21) in $\left[\mathrm{O}_{1}\right]$ and the inequality following $(38)$ in $\left[\mathrm{O}_{3}\right]$ ). (We shall present the short proof of (1.6) in the Appendix.) This answers a question raised by $\mathrm{Oh}\left[\mathrm{O}_{1}\right]$ after he found a technical error in an argument of Floer and Weinstein concerning (2.9) in [FW] (which is the reason that he required $V \in(V)_{a}$ even when $V$ is bounded).

Recently, Gui [G] pointed out that the nondegeneracy condition on critical points of $V$ in $\left[\mathrm{FW}, \mathrm{O}_{1}, \mathrm{O}_{3}\right]$ can be weakened.

\section{Section 2. Positive Ground States}

To study (1.3) and (1.4), following common practice, we define $E_{h}$ to be the Hilbert subspace of $H^{1}\left(\mathbb{R}^{n}\right)$ consisting of real-valued functions $v$ such that

$$
\|v\|_{E_{h}}^{2}=\int_{\mathbb{R}^{n}}\left(|\nabla v|^{2}+V_{h} v^{2}\right) d x<+\infty
$$

where $V_{h}(x)=V(h x)$. Since $\inf V>0$ (as we always assume), $E_{h}$ is imbedded continuously into $H^{1}\left(\mathbb{R}^{n}\right)$. We also define the energy functional associated with (1.4),

$$
I_{h}(v)=\frac{1}{2} \int_{\mathbb{R}^{n}}\left(|\nabla v|^{2}+V_{h} v^{2}\right) d x-\frac{1}{p+1} \int_{\mathbb{R}^{n}}|v|^{p+1} d x
$$


Then it is well-known (see, e.g. [DN]) that $I_{h}$ is well-defined on $E_{h}$, $I_{h} \in C^{1}\left(E_{h}, \mathbb{R}\right)$, and any critical point of $I_{h}$ gives a classical solution of (1.4). To study critical points of $I_{h}$, we further introduce

$$
M_{h}=\left\{\left.v \in E_{h} \backslash\{0\}\left|\int_{\mathbb{R}^{n}}\left(|\nabla v|^{2}+V_{h} v^{2}\right) d x=\int_{\mathbb{R}^{n}}\right| v\right|^{p+1} d x\right\}
$$

( $M_{h}$ is called the solution manifold because all $H^{1}\left(\mathbb{R}^{n}\right)$ solutions of (1.4) must belong to $M_{h}$ ),

$$
\Gamma_{h}=\left\{\eta \in C\left([0,1], E_{h}\right) \mid \eta(0)=0, \eta(1) \not \equiv 0, I_{h}(\eta(1)) \leqq 0\right\}
$$

and the mountain pass minimax value

$$
c_{h}=\inf _{\eta \in \Gamma_{h}} \max _{t \in[0,1]} I_{h}(\eta(t)) .
$$

Then for any $v \in E_{h} \backslash\{0\}$, there exists a unique $\theta>0$ such that

$$
I_{h}(\theta v)=\max _{t \geqq 0} I_{h}(t v), \quad \theta v \in M_{h} ;
$$

furthermore,

$$
0<c_{h}=\inf _{v \in M_{h}} I_{h}(v)=\inf _{v \in E_{h} \backslash\{0\}} \max _{t \geqq 0} I_{h}(t v)
$$

(see, e.g., [NT] for the case of Neumann problem).

As mentioned in the Introduction, under the condition

$$
\liminf _{x \rightarrow \infty} V(x)>V^{0} \equiv \inf V>0 .
$$

Rabinowitz [R] proved for small $h$ the existence of a classical solution $v_{h}$ of (1.4) with $I_{h}\left(v_{h}\right)=c_{h}$. In view of (2.3), $v_{h}$ is a minimizer of $I_{h}$ on $M_{h}$. We remark that each minimizer $v_{h}$ of $I_{h}$ on $M_{h}$ is of one sign. Indeed, by (2.2), there exists an $\theta>0$ such that $\theta\left|v_{h}\right| \in M_{h}$ and

$$
\begin{aligned}
\max _{t \geqq 0} I_{h}\left(t\left|v_{h}\right|\right) & =I_{h}\left(\theta\left|v_{h}\right|\right)=\left(\frac{1}{2}-\frac{1}{p+1}\right) \theta^{p+1} \int_{\mathbb{R}^{n}}\left|v_{h}\right|^{p+1} d x \\
& =\theta^{p+1} I_{h}\left(v_{h}\right)=\theta^{p+1} c_{h} .
\end{aligned}
$$

This and (2.3) imply $\theta \geqq 1$. But since

$$
\int_{\mathbb{R}^{n}}\left(|\nabla| v_{h}||^{2}+V_{h} v_{h}^{2}\right) d x \leqq \int_{\mathbb{R}^{n}}\left(\left|\nabla v_{h}\right|^{2}+V_{h} v_{h}^{2}\right) d x=\int_{\mathbb{R}^{n}}\left|v_{h}\right|^{p+1}
$$

and $\theta v_{h} \in M_{h}$, we have $\theta \leqq 1$. Thus $\theta=1$ and $\left|v_{h}\right| \in M_{h}$. Hence $\left|v_{h}\right|$ is also a minimizer of $I_{h}$ on $M_{h}$. A routine argument implies $\left|v_{h}\right|$ is a classical solution of (1.4). Now by the strong maximum principle, $\left|v_{h}\right|$ never vanish and hence $v_{h}$ is of one sign.

Throughout this section, we shall always assume $v_{h}$ is chosen to be positive (note $I_{h}$ is an even functional). Let

$$
u_{h}(x)=v_{h}\left(\frac{x}{h}\right)
$$


Then $u_{h}$ is a positive ground state of (1.3). Conversely, such a ground state $u_{h}$ of (1.3) corresponds to a counterpart of (1.4). In this section, if not stated otherwise, $u_{h}$ always stands for an arbitrary positive ground state of (1.3), $u_{h}$ and $v_{h}$ are always related by (2.5).

Theorem 2.1. Suppose (2.4) holds. For each sequence $h_{k}^{\prime} \rightarrow 0$, there exists a subsequence $\left\{h_{k}\right\}$ such that $u_{k} \equiv u_{h_{k}}$ concentrates at a global minimum point of $x_{0}$ of $V$ in the following sense: For each large $k>0, u_{k}$ has only one local (hence global) maximum point $x_{k}, x_{k} \rightarrow x_{0}$ as $k \rightarrow \infty$, and for any $\delta>0$ and large $k$,

$$
\begin{gathered}
\max _{\left|x-x_{0}\right| \leqq \delta} u_{k}(x)>\left(V^{0}\right)^{\frac{1}{p-1}}, \\
u_{k}(x) \leqq C\left|\frac{x-x_{k}}{h_{k}}\right|^{\frac{1-n}{2}} \exp \left(-\sqrt{V^{0}}\left|x-x_{k}\right| / h_{k}\right) \quad \text { for }\left|x-x_{0}\right| \geqq \delta,
\end{gathered}
$$

where $C$ is independent of $k, \delta, u_{k}$ and $V$ (but dependent of $V^{0}$ ).

Remark 1. If the global minimum point of $V$ is unique, then all ground states $u_{h}$ concentrate at that point as $h \rightarrow 0^{+}$. In general, $V\left(x_{h}\right) \rightarrow V^{0} \equiv \inf V$ as $h \rightarrow 0^{+}$, where $x_{h}$ is the local maximum point of $u_{k}$ whose uniqueness for small $h$ is assured by this theorem.

Remark 2. By the proof of this theorem presented below,

$$
u\left(h \cdot+x_{h}\right) \rightarrow u_{0}(\cdot) \quad \text { in } C_{\mathrm{loc}}^{2}\left(\mathbb{R}^{n}\right), L^{\infty}\left(\mathbb{R}^{n}\right) \text { and } H^{1}\left(\mathbb{R}^{n}\right), \quad \text { as } h \rightarrow 0^{+},
$$

where $u_{0}$ is the unique solution of (2.8) below. In particular,

$$
\left\|u_{h}\right\|_{L^{\infty}\left(\mathbb{R}^{n}\right)} \rightarrow\left\|u_{0}\right\|_{L^{\infty}\left(\mathbb{R}^{n}\right)}=u_{0}(0) \quad \text { as } h \rightarrow 0^{+} .
$$

The proof of Theorem 2.1 will be lengthy, but the basic idea is to compare $v_{h}$ to the positive solution $u_{0}$ of

$$
\Delta u-V^{0} u+u^{p}=0, \quad u>0, \quad u(\infty)=0, \quad u(0)=\max u .
$$

By [GNN], $u_{0}$ is radial, $u_{0}^{\prime}(r)<0$ for $r \neq 0$ and

$$
u_{0}(r),\left|u_{0}^{\prime}(r)\right| \leqq C r^{-\frac{(n-1)}{2}} e^{-\sqrt{V^{0} r}} .
$$

By $[\mathrm{K}], u_{0}$ is unique. Define $E^{0}, I^{0}(v)$ and $M^{0}$ by replacing $V_{h}$ by the constant $V^{0}$ in the definitions of $E_{h}, I_{h}(v)$ and $M_{h}$. Define $\Gamma^{0}$ and $c^{0}$ by modifying the definitions of $\Gamma_{h}$ and $c_{h}$ in the obvious way. Then (2.2) and (2.3) with the corresponding modifications hold true. Moreover, $0<c^{0}=I^{0}\left(u_{0}\right)$, and $u_{0}$ and its translations are the only positive critical points of the functional $I^{0}$ (by [GNN] and $[\mathrm{K}]$ ).

To prove Theorem 2.1, we shall need

Lemma 2.2. $\lim _{h \rightarrow 0^{+}} c_{h}=c^{0}$.

Remark. What we only need in proving this lemma is $V^{0}>0$ - no requirement on $\liminf _{x \rightarrow \infty} V$. 
Proof of Lemma 2.2. For any $R>0$, take a $\varphi_{R} \in C_{0}^{\infty}\left(\mathbb{R}^{n}\right)$ such that $\varphi_{R} \equiv 1$ on $B_{R}(0)=\{|x| \leqq R\}, \varphi_{R} \equiv 0$ in $B_{R+1}^{c}(0), 0 \leqq \varphi_{R} \leqq 1,\left|\nabla \varphi_{R}\right| \leqq c(n)$. Let $v_{R}=\varphi_{R} u_{0}$. Take a sequence $y_{k}$ such that $V\left(y_{k}\right) \rightarrow V^{0}$. Let $w(x)=v_{R}\left(x-\frac{y_{k}}{h}\right)$. Then there exists a unique $\theta>0$ such that $\theta w \in M_{h}$, i.e.,

$$
\begin{gathered}
\theta^{2} \int_{\mathbb{R}^{n}}\left(|\nabla w|^{2}+V(h x) w^{2}\right) d x=\theta^{p+1} \int_{\mathbb{R}^{n}} w^{p+1} d x, \\
\int_{\mathbb{R}^{n}}\left(\left|\nabla v_{R}\right|^{2}+V\left(h x+y_{k}\right) v_{R}^{2}\right) d x=\theta^{p-1} \int_{\mathbb{R}^{n}} v_{R}^{p+1} d x .
\end{gathered}
$$

Whence,

$$
\begin{aligned}
\theta^{p-1} & =\frac{\int_{\mathbb{R}^{n}}\left|\nabla v_{R}\right|^{2}+V^{0} v_{R}^{2}}{\int_{\mathbb{R}^{n}} v_{R}^{p+1}}+\frac{\int_{\mathbb{R}^{n}}\left(V\left(h x+y_{k}\right)-V^{0}\right) v_{R}^{2}}{\int_{\mathbb{R}^{n}} v_{R}^{p+1}} \\
& =I_{1}+I_{2}
\end{aligned}
$$

Since $u_{0}$ is a solution of (2.8), it is easy to see $I_{1} \rightarrow 1$ as $R \rightarrow \infty$. Also, for a fixed $R$, if we take a large $k$ so that $V\left(y_{k}\right)$ is close to $V^{0}$ and fix such a $k$, then $I_{2}$ is small as $h \rightarrow 0^{+}$. Thus when $R$ and $k$ are taken large and fixed, $\theta$ is close to 1 as $h \rightarrow 0^{+}$. Observe

$$
\begin{aligned}
c_{h} & =\inf _{M_{k}} I_{h}(v) \leqq I_{h}(\theta w) \\
& =\theta^{2}\left[I_{h}(w)+\frac{1-\theta^{p-1}}{p+1} \int_{\mathbb{R}^{n}} w^{p+1} d x\right] \\
& =\theta^{2}\left[I^{0}(w)+\frac{1}{2} \int_{\mathbb{R}^{n}}\left(V(h x)-V^{0}\right) w^{2} d x+\frac{1-\theta^{p-1}}{p+1} \int_{\mathbb{R}^{n}} w^{p+1} d x\right] \\
& =\theta^{2}\left[I^{0}\left(v_{R}\right)+\frac{1}{2} \int_{\mathbb{R}^{n}}\left(V\left(h x+y_{k}\right)-V^{0}\right) v_{R}^{2} d x+\frac{1-\theta^{p-1}}{p+1} \int_{\mathbb{R}^{n}} v_{R}^{p+1} d x\right] .
\end{aligned}
$$

Obviously $I^{0}\left(v_{R}\right) \rightarrow I^{0}\left(u_{0}\right)=c^{0}$ and $R \rightarrow \infty$. This and the property of $\theta$ discussed above imply that the last quantity in the above inequalities is close to $c^{0}$ if $R$ and $k$ are taken large and fixed, and then we let $h \rightarrow 0^{+}$. Thus lim $\sup _{h \rightarrow 0^{+}} c_{h} \leqq c^{0}$. On the other hand, since $I_{h}(v) \geqq I^{0}(v)$ for $v \in E_{h}$, we have $c_{h} \geqq c^{0}$. Now the desired conclusion follows.

Proof of Theorem 2.1. First, we observe that since

$$
\left(\frac{1}{2}-\frac{1}{p+1}\right) \int_{\mathbb{R}^{n}}\left(\left|\nabla v_{h}\right|^{2}+V_{h}(x) v_{h}^{2}\right) d x=I_{h}\left(v_{h}\right)=c_{h} \rightarrow c^{0}
$$

as $h \rightarrow 0^{+},\left\|v_{h}\right\|_{H^{1}\left(\mathbb{R}^{n}\right)}$ is bounded as $h \rightarrow 0^{+}$. 
Claim 1. There exists a sequence $\left\{y_{h}\right\}$ and positive constants $R$ and $\beta$ such that

$$
\liminf _{h \rightarrow 0^{+}} \int_{B_{R}\left(y_{h}\right)} v_{h}^{2}(x) d x \geqq \beta>0 .
$$

For otherwise, for any $R>0$, there exists a sequence $v_{k} \equiv v_{h_{k}}$ such that

$$
\lim _{k \rightarrow \infty} \sup _{y \in \mathbb{R}^{n}} \int_{B_{R}(y)} v_{k}^{2}(x) d x=0 .
$$

By Lemma $\mathrm{I} .1$ in $\left[\mathrm{L}_{2}\right]$ or Lemma 2.18 in $[\mathrm{CR}], v_{k} \rightarrow 0$ in $L^{q}\left(\mathbb{R}^{n}\right)$ for any $2<q<\frac{2 n}{(n-2)^{+}}$. This is impossible because

$$
\left(\frac{1}{2}-\frac{1}{p+1}\right) \int_{\mathbb{R}^{n}} v_{h}^{p+1} d x=c_{h} \rightarrow c^{0} \quad \text { as } h \rightarrow 0 .
$$

Claim 1 is proved.

Now let $w_{h}(x)=v_{h}\left(x+y_{h}\right)=u_{h}\left(h x+h y_{h}\right)$. Then by $(2.10)$,

$$
\liminf _{h \rightarrow 0^{+}} \int_{B_{R}(0)} w_{h}^{2}(x) d x \geqq \beta>0 .
$$

Furthermore,

$$
\begin{aligned}
& \Delta w_{h}-V\left(h x+h y_{h}\right) w_{h}+w_{h}^{p}=0, \quad w_{h}>0 \text { in } \mathbb{R}^{n}, \\
& c_{h}=\left(\frac{1}{2}-\frac{1}{p+1}\right) \int_{\mathbb{R}^{n}}\left(\left|\nabla w_{h}\right|^{2}+V\left(h x+h y_{h}\right) w_{h}^{2}\right) d x \\
& =\left(\frac{1}{2}-\frac{1}{p+1}\right) \int_{\mathbb{R}^{n}} w_{h}^{p+1} d x .
\end{aligned}
$$

Claim 2. $h y_{h}$ is bounded for small $h>0$. Otherewise, there exists a sequence $h_{m} \rightarrow 0^{+}$such that $h_{m} y_{h_{m}} \rightarrow \infty$. By (2.13) and Lemma 2.2, $w_{m} \equiv w_{k_{m}}$ is bounded in $H^{1}\left(\mathbb{R}^{n}\right)$. Hence by passing to a subsequence if necessary, $w_{m} \rightarrow w_{0} \geqq 0$ weakly in $H^{1}\left(\mathbb{R}^{n}\right)$, strongly in $L_{\mathrm{loc}}^{q}\left(\mathbb{R}^{n}\right)\left(2<q<\frac{2 n}{(n-2)^{+}}\right)$and a.e. in $\mathbb{R}^{n}$. By (2.11), $w_{0} \not \equiv 0$. So there exists a $\theta>0$ such that $\theta w_{0} \in M^{0}$. On the other hand, since

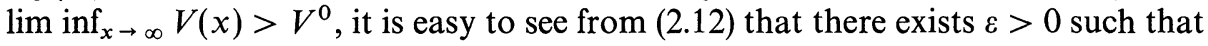

$$
\Delta w_{0}-\left(V^{0}+\varepsilon\right) w_{0}+w_{0}^{p} \geqq 0 \quad \text { in the } H^{-1}\left(\mathbb{R}^{n}\right) \text { sense } .
$$

In particular

$$
\int_{\mathbb{R}^{n}}\left(\left|\nabla w_{0}\right|^{2}+V^{0} w_{0}^{2}\right) d x<\int_{\mathbb{R}^{n}} w_{0}^{p+1} d x
$$


Thus $0<\theta<1$. Now, we have

$$
\begin{aligned}
c^{0} & =\inf _{M^{0}} I^{0}(v) \leqq I^{0}\left(\theta w_{0}\right) \\
& =\left(\frac{1}{2}-\frac{1}{p+1}\right) \int_{\mathbb{R}^{n}}\left(\theta w_{0}\right)^{p+1} d x \\
& <\left(\frac{1}{2}-\frac{1}{p+1}\right) \int_{\mathbb{R}^{n}} w_{0}^{p+1} d x \\
& \leqq \liminf _{m \rightarrow \infty}\left(\frac{1}{2}-\frac{1}{p+1}\right) \int_{\mathbb{R}^{n}} w_{m}^{p+1} d x \quad \text { (Fatou's Lemma) } \\
& =\lim _{m \rightarrow \infty} I_{h_{m}}\left(w_{m}\right)=c^{0} \quad((2.13) \text { and Lemma 2.2). }
\end{aligned}
$$

This is impossible and hence Claim 2 is proved.

Now for any sequence $h_{k}^{\prime} \rightarrow 0$, there exists a subsequence $h_{k}$ such that $\bar{x}_{k} \equiv h_{k} y_{h_{k}} \rightarrow x_{0}, w_{k} \equiv w_{h_{k}} \rightarrow w_{0} \geqq 0, \not \equiv 0$, weakly in $H^{1}\left(\mathbb{R}^{n}\right)$ and a.e. in $\mathbb{R}^{n}$.

Claim 3. $x_{0}$ is a global minimum point of $V$.

By applying the elliptic regularity theory to (2.12), we have $w_{k} \rightarrow w_{0}$ in $C_{\text {loc }}^{2}\left(\mathbb{R}^{n}\right)$ and

$$
\Delta w_{0}-V\left(x_{0}\right) w_{0}+w_{0}^{p}=0, \quad x \in \mathbb{R}^{n} .
$$

So

$$
\int_{\mathbb{R}^{n}}\left(\left|\nabla w_{0}\right|^{2}+V^{0} w_{0}^{2}\right) d x \leqq \int_{\mathbb{R}^{n}}\left(\left|\nabla w_{0}\right|^{2}+V\left(x_{0}\right) w_{0}^{2}\right) d x=\int_{\mathbb{R}^{n}} w_{0}^{p+1} d x
$$

Therefore, there exists a $0<\theta \leqq 1$ such that $\theta w_{0} \in M^{0}$. Now

$$
\begin{aligned}
c^{0} & =\lim _{k \rightarrow \infty} c_{h_{k}}=\lim _{k \rightarrow \infty}\left(\frac{1}{2}-\frac{1}{p+1}\right) \int_{\mathbb{R}^{n}} w_{k}^{p+1} d x \\
& \geqq\left(\frac{1}{2}-\frac{1}{p+1}\right) \int_{\mathbb{R}^{n}} w_{0}^{p+1} d x \\
& \geqq\left(\frac{1}{2}-\frac{1}{p+1}\right) \int_{\mathbb{R}^{n}}\left(\theta w_{0}\right)^{p+1} d x \\
& =I^{0}\left(\theta w_{0}\right) \geqq \inf _{M^{0}} I^{0}(v) \geqq c^{0} .
\end{aligned}
$$

Thus $\theta=1$ and hence $V\left(x^{0}\right)=V^{0}(=\inf V)$. The proof of Claim 3 is complete. 
Now observe

$$
\begin{aligned}
\int_{\mathbb{R}^{n}}\left|\nabla w_{0}\right|^{2}+V\left(x_{0}\right) w_{0}^{2} d x & \leqq \liminf _{k \rightarrow \infty} \int_{\mathbb{R}^{n}}\left(\left|\nabla w_{k}\right|^{2}+V\left(x_{0}\right) w_{k}^{2}\right) d x \\
& \leqq \limsup _{k \rightarrow \infty} \int_{\mathbb{R}^{n}}\left(\left|\nabla w_{k}\right|^{2}+V\left(x_{0}\right) w_{k}^{2}\right) d x \\
& \leqq \limsup _{k \rightarrow \infty} \int_{\mathbb{R}^{n}}\left(\left|\nabla w_{k}\right|^{2}+V\left(h_{k} x+\bar{x}_{k}\right) w_{k}^{2}\right) d x
\end{aligned}
$$

$$
\begin{aligned}
& =\lim _{k \rightarrow \infty} \frac{c_{h_{k}}}{\left(\frac{1}{2}-\frac{1}{p+1}\right)}=\frac{c^{0}}{\left(\frac{1}{2}-\frac{1}{p+1}\right)} \\
& =\int_{\mathbb{R}^{n}}\left(\left|\nabla w_{0}\right|^{2}+V\left(x_{0}\right) w_{0}^{2}\right) d x .
\end{aligned}
$$

Thus $\int_{\mathbb{R}^{n}}\left|\nabla w_{k}\right|^{2}+V\left(x_{0}\right) w_{k}^{2} d x \rightarrow \int_{\mathbb{R}^{n}}\left|\nabla w_{0}\right|^{2}+V\left(x_{0}\right) w_{0}^{2}$ and hence $w_{k} \rightarrow w_{0}$ strongly in $H^{1}\left(\mathbb{R}^{n}\right)$. In particular,

$$
\int_{|x| \geqq R} w_{k}^{2 *} d x \rightarrow 0 \text { as } R \rightarrow \infty \text { uniformly w.r.t. } k,
$$

where $2^{*}=\frac{2 n}{n-2}$ if $n \geqq 3$, and an arbitrary large number if $n=1,2$. Note $w_{k}$ is a subsolution of $\Delta u+c(x) u=0$ with $c(x)=w_{k}^{p-1}$. By the one-sided Harnack inequality (see $[\mathrm{T}]$ ), we have

$$
\max _{B_{1}(Q)} w_{k} \leqq c\left(\int_{B_{2}(Q)} w_{k}^{2 *} d x\right)^{\frac{1}{2 *}}
$$

where $Q$ is an arbitrary point in $\mathbb{R}^{n}, c$ is a constant depending only on $n$ and the bound of $\left\|w_{k}\right\|_{L^{2 *}\left(B_{2}(Q)\right)}$. Thus by (2.15),

$$
w_{k}(x) \rightarrow 0 \text { as }|x| \rightarrow \infty \text { uniformly w.r.t. } k \text {. }
$$

Hence $u_{k}(x) \equiv u_{h_{k}}(x)=w_{k}\left(\frac{x-\bar{x}_{k}}{h_{k}}\right)$ decays to zero uniformly for $x$ outside any fixed neighborhood of $x_{0}$ as $k \rightarrow \infty$. Let $x_{k}$ be a local maximum point of $u_{k}$. By (1.3) and the strong maximum principle, $u_{k}\left(x_{k}\right)>\left(V^{0}\right)^{\frac{1}{p-1}}$. Therefore $x_{k} \rightarrow x_{0}$ as $k \rightarrow \infty$. Then

It remains to show the uniqueness of $x_{k}$ and (2.7). Let $\bar{w}_{k}(x)=u_{k}\left(h_{k} x+x_{k}\right)$.

$$
\Delta \bar{w}_{k}(x)-V\left(h_{k} x+x_{k}\right) \bar{w}_{k}+\bar{w}_{k}^{p}=0, \quad \bar{w}_{k}>0 \quad \text { in } \mathbb{R}^{n},
$$

$O$ is a critical point of $\bar{w}_{k}$ and $\bar{w}_{k}(0)>\left(V^{0}\right)^{\frac{1}{p-1}}$. The arguments similar to those concerning $w_{k}$ presented above show that after passing to a subsequence of $\left\{\bar{w}_{k}\right\}$, 
$\bar{w}_{k} \rightarrow w_{0}$ in $C_{\text {loc }}^{2}\left(\mathbb{R}^{n}\right), H^{1}\left(\mathbb{R}^{n}\right)$ and $L^{\infty}\left(\mathbb{R}^{n}\right)$ where $w_{0} \not \equiv 0$ satisfies (2.14); furthermore, $\bar{w}_{k} \rightarrow 0$ as $x \rightarrow \infty$ uniformly w.r.t. $k$. Since $O$ is a critical point of $\bar{w}_{k}$, it is also a critical point of $w_{0}$. By [GNN], $w_{0}$ is spherically symmetric about some point $P$, and $w_{0}^{\prime}(s)<0$ for $0 \neq s=|x-P|$. Thus $P=$ the origin, i.e., $w_{0}$ is radial. Since $\bar{w}_{k}$ decays to zero in $x$ uniformly w.r.t. $k$, so does $w_{0}$. Now Kwong's result $[\mathrm{K}]$ says $w_{0} \equiv u_{0}($ see $(2.8))$.

To show the uniqueness of the local maximum point of $u_{k}$, it suffices to do so for $\bar{w}_{k}$. Observe at each local maximum point of $\bar{w}_{k}, \bar{w}_{k}>\left(V^{0}\right)^{\frac{1}{p-1}}$. Since $\bar{w}_{k}$ decays to zero uniformly w.r.t. $k$ as $x \rightarrow \infty$, all the local maximum points of $\bar{w}_{k}$ stay in a finite ball in $\mathbb{R}^{n}$. Since $\bar{w}_{k} \rightarrow w_{0}=u_{0}$ in $C_{\text {loc }}^{2}\left(\mathbb{R}^{n}\right)$ and $O$ is the only critical point of $u_{0}$, these points must approach the origin and hence stay in a small ball $B_{\varepsilon}(0)$ as $k \rightarrow \infty$. As can be easily seen, we can take $\varepsilon$ so small that $u_{0}^{\prime \prime}(r)<0$ for $0 \leqq r \leqq \varepsilon$. Now by Lemma 4.2 in [NT], for large $k, \bar{w}_{k}$ has no critical points other than the origin.

To show (2.7), we recall that $\bar{w}_{k}(x)$ decays to zero at $x=\infty$ uniformly w.r.t. $k$. Applying the argument in the proof of Proposition 4.1 in [GNN] (see also Kato $[\mathrm{Ka}])$, we have

$$
\bar{w}_{k}(x) \leqq C|x|^{\frac{1-n}{2}} \exp \left(-\sqrt{V^{0}}|x|\right) \quad \text { for }|x| \geqq 1 \text { and large } k,
$$

where $C$ is independent of $k, \bar{w}_{k}$ and $V$ (but dependent of $V^{0}$ ). This implies (2.7) .

Our next result deals with the situation when we do not know if $\liminf _{x \rightarrow \infty} V(x)>\inf V$. In this case, (1.3) may or may not have a ground state: in the trivial case $V \equiv$ constant $>0,(1.3)$ has infinitely many positive ground states, while when for some direction, the directional derivative of $V$ is nonnegative but not identically zero, then it is possible to prove by integration by parts that (1.3) does not even possess an $H^{1}\left(\mathbb{R}^{n}\right)$ solution $\not \equiv 0$ (see the proof of Theorem 3.1 in Sect. 3). However, we have

Theorem 2.3. Suppose there exists a sequence $h_{k} \rightarrow 0^{+}$such that (1.3) with $h=h_{k}$ has a positive ground state $u_{k}$ and $u_{k}$ has a local maximum point $x_{k}$ which converges to some point $x_{0}$ as $k \rightarrow \infty$. Then $x_{0}$ must be a global minimum point of $V, x_{k}$ is the only local (hence global) maximum point of $u_{k}$ for $k$ large. Moreover, (2.6) and (2.7) hold true.

Proof. Since the details of this proof are similar to those of the proof of Theorem 2.1, we shall only sketch the main steps. Let $w_{k}(x)=u_{k}\left(h_{k} x+x_{k}\right)$.

Step 1. Show $x_{0}$ is a global minimum point of $V$ by arguing as in the proof of Claim 3 in the proof of Theorem 2.1.

Step 2. By using the conclusion in Step 1 , show $w_{k} \rightarrow u_{0}$ in $H^{1}\left(\mathbb{R}^{n}\right)$ as $k \rightarrow \infty$, where $u_{0}$ satisfies $(2.8)$.

Step 3. By using the conclusion in Step 2 and the one-sided Harnack inequality, show $w_{k}$ decays to zero at $x=\infty$ uniformly w.r.t. $k$. Then (2.7) follows.

Step 4. Show the uniqueness of $x_{k}$. 
In the remaining part of this section, we deal with the case when the potential $V$ is radial and is bounded below from zero. Define $E_{h}^{r}$ and $M_{h}^{r}$ to be the subset of $E_{h}$ and $M_{h}$ consisting of radial functions. Define

$$
c_{h}^{r}=\inf _{M_{h}^{r}} I_{h}(v) .
$$

It is routine to see $c_{h}^{r}>0$. Then

Proposition 2.4. Assume $n \geqq 2$. Then for each $h>0, c_{h}^{r}$ is assumed by a minimizer $v_{h}$ of $I_{h}$ over $M_{h}^{r}$.

Remark. As we mentioned in Sect. 1, this follows essentially from a general result in [DN]. However, in the present case, we can prove this result by showing that a minimizing sequence converges to some $v_{h} \in M_{h}^{r}$ by virtue of the standard fact that $E_{h}^{r} \subseteq L^{q}$ compactly with $2<q<\frac{2 n}{(n-2)^{+}}$. An alternative is to apply the Mountain Pass type argument as in [R] and the well-known Radial Lemma of Strauss.

It is easy to see $v_{h}$ is also a (radial) solution of (1.4). As before, we can show that $v_{h}$ is of one sign and hence we shall always assume that $v_{h}>0$. Let $u_{h}(r)=v_{h}\left(\frac{r}{h}\right)$ $(r=|x|)$. Then $u_{h}$ is a positive radial solution of (1.3) with least energy (1.5) among all nontrivial radial $H^{1}\left(\mathbb{R}^{n}\right)$ solutions. Conversely, each such radial ground state of (1.3) corresponds to a $v_{h}$ in the statement of Proposition 2.4.

Theorem 2.5. Suppose $n \geqq 2$ and $V$ is radial. Let $u_{h}$ be a positive radial ground state of (1.3) (whose existence is assured by Proposition 2.4). Then $u_{h}$ concentrates at the origin in the following sense: For small $h>0, u_{h}$ has only one local (hence global) maximum point $x_{h}, x_{h} \rightarrow 0$ as $h \rightarrow 0$; moreover (2.6) and (2.7) with $x_{0}=x_{k}=0, h_{k}=h$ and $u_{k}=u_{h}$ hold true.

Remark. If $V(0)$ is not a global minimum of $V$, by combining Theorem 2.3 and Theorem 2.5, we see that a ground state, i.e., least energy solution of (1.3), if any, must not be radial for small $h>0$.

Proof of Theorem 2.5. By the proof of Lemma 2.2, $c_{h}^{r}$ is bounded for $1 \geqq h>0$. Thus $\left\|v_{h}\right\|_{H^{1}\left(\mathbb{R}^{n}\right)}$ is also bounded for $1 \geqq h>0$. Recall the Radial Lemma of Strauss [S]:

$$
|u(r)| \leqq C r^{\frac{1-n}{2}}\|u\|_{H^{1}\left(\mathbb{R}^{n}\right)} \text { for } r \geqq 1,
$$

where $C$ depends on $n$. Using this, we have that $v_{h}$ decays at $r=\infty$ uniformly w.r.t. small $h>0$. Now applying the arguments in the proof of Proposition 4.1 in [GNN] to (1.4), one has

$$
v_{h}(r) \leqq C r^{\frac{1-n}{2}} \exp \left(-\sqrt{V^{0}} r\right) \text { for } r \geqq 1,
$$

from which (2.7) with the modification follows. 
If $x_{h}$ is a local maximum point of $u_{h}$, then by the maximum principle, $u_{h}\left(x_{h}\right)>\left(V^{0}\right)^{\frac{1}{p-1}}$. This and (2.7) imply $x_{h} \rightarrow 0$ as $h \rightarrow 0$. To show $x_{h}$ is unique, observe that since $\left\|v_{h}\right\|_{H^{1}\left(\mathbb{R}^{n}\right)}$ is bounded for small $h$ and $v_{h}$ satisfies (1.4), each sequence of $\left\{v_{h}\right\}$ contains a subsequence $\left\{v_{k}\right\}$ such that $v_{k} \rightarrow$ some $\bar{u}_{0}$ weakly in $H^{1}\left(\mathbb{R}^{n}\right)$ and strongly in $C_{\text {loc }}^{2}\left(\mathbb{R}^{n}\right)$, where $\bar{u}_{0}$ is a radial solution of

$$
\Delta u-V(0) u-u^{p}=0, \quad u>0, \quad u(\infty)=0 .
$$

By Kwong $[\mathrm{K}], \bar{u}_{0}$ is unique. Thus $v_{h} \rightarrow \bar{u}_{0}$ in $C_{\mathrm{loc}}^{2}\left(\mathbb{R}^{n}\right)$ and $L^{\infty}\left(\mathbb{R}^{n}\right)$ as $h \rightarrow 0^{+}$. Now we can show the uniqueness of $x_{h}$ as in the proof of Theorem 2.1.

Remark. By the proof above, $\left\|u_{h}\right\|_{L^{\infty}\left(\mathbb{R}^{n}\right)} \rightarrow\left\|\bar{u}_{0}\right\|_{L^{\infty}}=\bar{u}_{0}(0)$, where $\bar{u}_{0}$ is the unique radial solution of (2.18).

\section{Section 3. Positive Bound States}

The purpose of this section is to show that a point at which a sequence of positive bound states concentrates must be a critical point of $V$. Recall Floer, Weinstein and Oh have obtained results converse to this. Recall also by a bound state, we mean an $H^{1}\left(\mathbb{R}^{n}\right)$ solution of (1.3) with finite energy (1.5). (It is well-known that a (positive) bound state decays exponentially at $\infty$, as may be proved by the one-sided Harnack inequality and then using a result of [GNN].)

Theorem 3.1. Assume $|\nabla V(x)|=O\left(e^{a|x|}\right)$ at $x=\infty$ for some $a>0$. Let $u_{k} \equiv u_{h_{k}}$ be a sequence of positive bound states of (1.3) with $h=h_{k}$. Suppose $u_{k}$ concentrates at a point $x_{0}$ in the following sense: $\forall \varepsilon>0, \exists$ constants $R$ and $K>0$ such that

$$
u_{k}(x) \leqq \varepsilon \quad \text { for } k \geqq K \text { and }\left|x-x_{0}\right| \geqq h_{k} R .
$$

Then $\nabla V\left(x_{0}\right)=0$. Moreover, for large $k, u_{k}$ has only one local maximum point $x_{k}$, $x_{k} \rightarrow x_{0}$ as $k \rightarrow \infty$, and (2.6)-(2.7) with $x_{k}$ replaced by $x_{0}$ hold true.

Remark 1. It is possible to check the solutions obtained by Floer, Weinstein and $\mathrm{Oh}\left[\mathrm{FW}, \mathrm{O}_{1}\right]$ satisfy (3.1).

Remark 2. We suspect that the condition $|\nabla V(x)|=O\left(e^{a|x|}\right)$ could be removed.

Proof of Theorem 3.1. We shall break up this proof into 3 steps; In Step 1, we show $\left\|u_{k}\right\|_{L^{\infty}\left(\mathbb{R}^{n}\right)}$ is bounded. In Step 2, we show the second part of this theorem; In Step 3 , we prove $\nabla V\left(x_{0}\right)=0$.

Step 1. Suppose $\exists$ a sequence $h_{m} \rightarrow 0$ so that the $L^{\infty}\left(\mathbb{R}^{n}\right)$ norm of $u_{m}=u_{h_{m}}$ tends to $\infty$ as $m \rightarrow \infty$. Let $\alpha_{m}=\max u_{m}$ and $\beta_{m}=\alpha_{m}^{-(p-1) / 2}$. Define

$$
v_{m}(x)=\frac{1}{\alpha_{m}} u_{m}\left(x_{m}+h_{m} \beta_{m} x\right),
$$

where $x_{m}$ is a global maximum point of $u_{m}$. Then

$$
\Delta v_{m}-\beta_{m}^{2} V\left(x_{m}+h_{m} \beta_{m} x\right) v_{m}+v_{m}^{p}=0, \quad x \in \mathbb{R}^{n},
$$


and

$$
v_{m}(0)=1, \quad 0 \leqq v_{m} \leqq 1
$$

Therefore, by the elliptic regularity theory, we have

$$
v_{m} \rightarrow v_{0} \text { is } C_{\mathrm{loc}}^{2}\left(\mathbb{R}^{n}\right) \text { as } m \rightarrow \infty,
$$

where

$$
\Delta v_{0}+v_{0}^{p}=0 \quad \text { in } \mathbb{R}^{n}, \quad v_{0}(0)=1 .
$$

This is impossible according to [CL] or [CGS]. Step 1 is finished.

Step 2. Let $w_{k}(x)=u_{k}\left(x_{0}+h_{k} x\right)$. Then

$$
\Delta w_{k}-V\left(x_{0}+h_{k} x\right) w_{k}+w_{k}^{p}=0, \quad x \in \mathbb{R}^{n},
$$

and by (3.1), $w_{k}$ decays to zero uniformly w.r.t. $k$. Now, as before, by the arguments in the proof of Proposition 4.1 in [GNN],

$$
w_{k}(x) \leqq C|x|^{\frac{1-n}{2}} \exp \left(-\sqrt{V^{0}}|x|\right) \text { for }|x| \geqq 1 .
$$

This implies (2.7) with $x_{k}$ there replaced by $x_{0}$. Observe by the conclusion in Step 1 and the elliptic regularity, for any subsequence of $\left\{w_{k}\right\}$, there exists a subsequence $\left\{w_{k}^{\prime}\right\}$ of the subsequence such that $w_{k}^{\prime} \rightarrow$ some $w_{0}$ in $C_{\text {loc }}^{2}\left(\mathbb{R}^{n}\right)$, where $w_{0}$ satisfies

$$
\Delta w_{0}-V\left(x_{0}\right) w_{0}+w_{0}^{p}=0, \quad x \in \mathbb{R}^{n} .
$$

By (3.3) and the fact that local maximum values of $w_{k}$ are larger than $\left(V^{0}\right)^{\frac{1}{p-1}}$ we see local maximum points of $w_{k}$ must stay in a fixed ball for all $k$. This and the fact that $w_{k}^{\prime} \rightarrow w_{0}$ in $C_{\text {loc }}^{2}\left(\mathbb{R}^{n}\right)$ imply $w_{0} \not \equiv 0$ and hence positive by the strong maximum principle. Now we can show the uniqueness of local maximum point of $w_{k}^{\prime}$ for $k$ large as in the proof of Theorem 2.1. From this the uniqueness of $x_{k}$ follows. By (3.1), $x_{k} \rightarrow x_{0}$. Equation (2.6) follows from the strong maximum principle. Now Step 2 is complete.

Step 3. Without loss of any generality, assume $w_{k} \rightarrow w_{0}$ in $C_{\text {loc }}^{2}\left(\mathbb{R}^{n}\right)$. Multiplying (3.2) by $\nabla w_{k}$ and integrating on $B_{R}(0)$, we have

$$
\begin{gathered}
0=\int_{B_{R}} \Delta w_{k} \nabla w_{k}-\frac{1}{2} \nabla\left(V\left(x_{0}+h_{k} x\right) w_{k}^{2}\right) \\
+\frac{1}{2} h_{k} \nabla V\left(x_{0}+h_{k} x\right) w_{k}^{2}+\frac{\nabla w_{k}^{p+1}}{p+1} d x \\
\frac{h_{k}}{2} \int_{B_{R}} \nabla V\left(x_{0}+h_{k} x\right) w_{k}^{2} d x=\int_{\partial B_{R}}\left(\frac{1}{2} V\left(x_{0}+h_{k} x\right) w_{k}^{2} v\right. \\
\left.-\frac{1}{p+1} w_{k}^{p+1} v\right) d s-\int_{B_{R}} \Delta w_{k} \nabla w_{k} d x
\end{gathered}
$$


where $v$ is the exterior normal field on $\partial B_{R}$. We compute the second integral on the right-hand side as follows (write $w_{k}$ as $w$ ):

$$
\begin{aligned}
\int_{B_{R}} \Delta w \frac{\partial w}{\partial x_{i}} d x & =\int_{B}\left(\operatorname{div}\left(\nabla w \frac{\partial w}{\partial x_{i}}\right)-\nabla w \frac{\partial}{\partial x_{i}} \nabla w\right) d x \\
& =\int_{\partial B}\left(\frac{\partial w}{\partial x_{i}} \frac{\partial w}{\partial v}-v_{i}|\nabla w|^{2} / 2\right) d S .
\end{aligned}
$$

Therefore (3.5) becomes

$$
\begin{aligned}
\frac{h_{k}}{2} \int_{B_{R}} \nabla V\left(x_{0}+h_{k} x\right) w_{k}^{2} d x= & \int_{\partial B_{R}}\left(\frac{1}{2} V\left(x_{0}+h_{k} x\right) w_{k}^{2} v-\frac{1}{p+1} w_{k}^{p+1} v\right. \\
& \left.-\nabla w_{k} \frac{\partial w_{k}}{\partial v}+v\left|\nabla w_{k}\right|^{2} / 2\right) d S \equiv I_{R}
\end{aligned}
$$

Observe

$$
\begin{aligned}
\int_{0}^{\infty}\left|I_{R}\right| d R & \leqq \int_{0}^{\infty} d R \int_{\partial B_{R}}\left(\frac{3}{2}\left(\left|\nabla w_{k}\right|^{2}+V\left(x_{0}+h_{k} x\right)\right) w_{k}^{2}+\frac{1}{p+1} w_{k}^{p+1}\right) d S \\
& \leqq \frac{3}{2} \int_{\mathbb{R}^{n}}\left(\left(\left|\nabla w_{k}\right|^{2}+V\left(x_{0}+h_{k} x\right) w_{k}^{2}+w_{k}^{p+1}\right) d x\right. \\
& <+\infty \text { for each } k,
\end{aligned}
$$

by the assumption that $u_{k}$ is a bound state. Thus for each fixed $k$ there exists a sequence $R_{m} \rightarrow \infty$ such that $I_{R_{m}} \rightarrow 0$ as $m \rightarrow \infty$. Now letting $R=R_{m} \rightarrow \infty$ in (3.6), by virtue of (3.3), the growth condition on $|\nabla V|$ and the Dominated Convergence Theorem, we have

$$
\int_{\mathbb{R}^{n}} \nabla V\left(x_{0}+h_{k} x\right) w_{k}^{2} d x=0 .
$$

Now letting $h_{k} \rightarrow 0$ and by the Dominated Convergence Theorem again, we have

$$
\int_{\mathbb{R}^{n}} \nabla V\left(x_{0}\right) w_{0}^{2} d x=0 .
$$

Thus $\nabla V\left(x_{0}\right)=0$.

\#

Remark. Equation (3.6) may be deduced from the dilated Pohozaev identity which appeared in, e.g., [H].

\section{Appendix}

We shall prove (1.6) when $V$ is bounded. Let $\bar{V}(x)=V(h x)-E$. For $u \in H^{2}\left(\mathbb{R}^{n}\right)$, let

$$
f=-\Delta u+\bar{V}(x) u \text {. }
$$


Multiplying (A.1) by $u$ and integrating on $\mathbb{R}^{n}$, we have

$$
\int_{\mathbb{R}^{n}}|\nabla u|^{2}+\bar{V}(x) u^{2} d x=\int_{\mathbb{R}^{n}} f u d x \leqq\|f\|_{L^{2}\left(\mathbb{R}^{n}\right)}\|u\|_{L^{2}\left(\mathbb{R}^{n}\right)} .
$$

Recalling $\bar{V} \geqq \delta>0$, we then have

$$
\|u\|_{L^{2}\left(\mathbb{R}^{n}\right)} \leqq \frac{1}{\delta}\|f\|_{L^{2}\left(\mathbb{R}^{n}\right)}
$$

By this and (A.1), we obtain

$$
\begin{aligned}
\|\Delta u\|_{L^{2}\left(\mathbb{R}^{n}\right)} & =\|f-\bar{V} u\|_{L^{2}\left(\mathbb{R}^{n}\right)} \leqq\|f\|_{L^{2}\left(\mathbb{R}^{n}\right)}+\|\bar{V}\|_{L^{\infty}\left(\mathbb{R}^{n}\right)}\|u\|_{L^{2}\left(\mathbb{R}^{n}\right)} \\
& \leqq\left(1+\|\bar{V}\|_{L^{\infty}\left(\mathbb{R}^{n}\right)} / \delta\right)\|f\|_{L^{2}\left(\mathbb{R}^{n}\right)} .
\end{aligned}
$$

So

$$
\begin{aligned}
\|u\|_{H^{2}\left(\mathbb{R}^{n}\right)} & =\|u\|_{L^{2}\left(\mathbb{R}^{n}\right)}+\|\Delta u\|_{L^{2}\left(\mathbb{R}^{n}\right)} \\
& \leqq\left(1+\left(\|\bar{V}\|_{L^{\infty}\left(\mathbb{R}^{n}\right)}+1\right) / \delta\right)\|f\|_{L^{2}\left(\mathbb{R}^{n}\right)} .
\end{aligned}
$$

Choosing $\lambda$ in the obvious way, we obtain (1.6). Note the dependence of $\lambda$ on $\|\bar{V}\|_{L^{\infty}\left(\mathbb{R}^{n}\right)}$ does not change the validity of the arguments in $[\mathrm{FW}],\left[\mathrm{O}_{1}\right]$ and $\left[\mathrm{O}_{3}\right]$.

Acknowledgement. We wish to thank Wei-Ming Ni for several helpful conversations. We are also grateful to the referee for many helpful suggestions concerning the exposition of this paper.

\section{References}

[CGS] Cafferelli, L., Gidas, B., Spruck, J.: Asymptotic symmetry and local behavior of semilinear elliptic equations with critical Sobolev growth. Comm. Pure Appl. Math. 42, 271-297 (1989)

[CL] Chen, W., Li, C.: Classification of solutions of some semilinear elliptic equations. Duke Math. J. 63, No. 3, 615-622 (1991)

[CR] Coti Zelati, V., Rabinowitz, P.H.: Homoclinic type solutions for a semilinear elliptic PDE on $\mathbb{R}^{n}$. Preprint

[DN] Ding, W.-Y., Ni, W.-M.: On the existence of positive entire solutions of a semilinear elliptic equation. Arch. Rational Mech. Anal. 91, 283-308 (1986)

[FW] Floer, A., Weinstein, A.: Nonspreading wave packets for the cubic Schrödinger equation with a bounded potential. J. Funct. Anal. 69, 397-408 (1986)

[G] Changfeng Gui: A remark on nonlinear Schrödinger equations. Preprint

[GNN] Gidas, B., Ni, W.-M., Nirenberg, L.: Symmetry of positive solutions of nonlinear elliptic equations in $\mathbb{R}^{n}$. Adv. Math. Suppl. Stud. 7A, Math. Anal. Appl. Part A, 369-402 (1981)

[GT] Gilbarg, D., Trudinger, N.: Elliptic Partial Differential Equations of Second Order. 2nd ed., New York, Berlin: Springer 1983

[H] Han, Z.-C.: Asymptotic approach to singular solutions for nonlinear elliptic equations involving critical Sobolev exponent. Analyse Nonlin. 8, 159-174 (1991)

[Ka] Kato, T.: Growth properties of solutions of the reduced wave equation with a variable coefficient. Comm. Pure Appl. Math. 12, 403-425 (1959)

[K] Kwong, M.: Uniqueness of positive solutions of $\Delta u-u+u^{p}=0$ in $\mathbb{R}^{n}$. Arch. Rational Mech. Anal. 105, 243-266 (1989)

$\left[\mathrm{L}_{1}\right]$ Lions, P.L.: On positive solutions of semilinear elliptic equations in unbounded domains. Nonlinear Diffusion Equations and their Equilibrium States, Vol. II (W.-M. Ni, L.A. Peletier, J. Serrin, eds.), MSRI Publications 13, 85-122 (1988) 
$\left[\mathrm{L}_{2}\right]$ Lions, P.L.: The concentration compactness principle in the calculus of variations. The locally compact case, Part 2 . Analyse Nonlin. 1, 223-283 (1984)

[MW] Mawhim, J., Willem, M.: Critical Point Theory and Hamiltonian Systems. Berlin, Heidelberg, New York: Springer 1989

[N] Ni, W.-M.: Some aspects of semilinear elliptic equations in $\mathbb{R}^{n}$. Nonlinear Diffusion Equations and their Equilibrium States, Vol. II (W.-M. Ni, L.A. Peletier, J. Serrin, eds.), MSRI Publications 13, 171-205 (1988)

[NT] Ni, W.-M., Takagi, I.: On the shape of least-energy solutions to a semilinear Neumann problem. Comm. Pure Appl. Math. XLIV, 819-851 (1991)

$\left[\mathrm{O}_{1}\right]$ Oh, Y.-G.: Existence of semi-classical bound states of nonlinear Schrödinger equations with potential of the class $(V)_{a}$. Comm. Partial Diff. Eq. 13, 1499-1519 (1988)

$\left[\mathrm{O}_{2}\right]$ Oh, Y.-G.: Corrections to Existence of semiclassical bound states of nonlinear Schrödinger equations with potentials of the class $(V)_{a}$. Comm. Partial Diff. Eq. 14, 833-834 (1989)

$\left[\mathrm{O}_{3}\right]$ Oh, Y.-G.: On positive multi-lump bound states of nonlinear Schrödinger equations under multiple well potential. Commun. Math. Phys. 131, 223-253 (1990)

[R] Rabinowitz, P.H.: On a class of nonlinear Schrödinger equations. to appear in ZAMP

[S] Strauss, W.A.: Existence of solitary waves in higher dimensions. Commun. Math. Phys. 55, 149-162 (1977)

[T] Trudinger, N.S.: On Harnack type inequalities and their applications to quasilinear elliptic equations. Comm. Pure Appl. Math. 20, 721-747 (1967)

Communicated by A. Jaffe 